\title{
Needle localization of small pulmonary nodules: Lessons learned
}

Patricia A. Thistlethwaite, MD, PhD, ${ }^{\mathrm{a}}$ Jonathan R. Gower, MD, ${ }^{\mathrm{a}}$ Moises Hernandez, MD, ${ }^{\mathrm{a}}$ Yu Zhang, MD, PhD, ${ }^{a}$ Andrew C. Picel, MD, ${ }^{b}$ and Anne C. Roberts, $\mathrm{MD}^{\mathrm{b}}$

\section{ABSTRACT}

Background: Lung nodules that are small and deep within lung parenchyma, and have semisolid characteristics are often challenging to localize with videoassisted thoracoscopic surgery (VATS). We describe our cumulative experience using needle localization of small nodules before surgical resection. We report procedural tips, operative results, and lessons learned over time.

Methods: A retrospective review of all needle localization cases between July 1, 2006, and December 30, 2016, at a single institution was performed. A total of 253 patients who underwent needle localization of lung nodules ranging from 0.6 to $1.2 \mathrm{~cm}$ before operation were enrolled. Nodules were localized by placing two 20-gauge Hawkins III coaxial needles from different trajectories with tips adjacent to the nodule, injection of 0.3 to $0.6 \mathrm{~mL}$ of methylene blue, and deployment of 2 hookwires, under computed tomography guidance. Patients then underwent VATS wedge resection for diagnosis, followed by anatomic resection for lung carcinoma. Procedural and perioperative outcomes were assessed.

Results: Needle localization was successful in 245 patients $(96.8 \%)$. Failures included both wires falling out of lung parenchyma before operation (5 patients), wire migration ( 2 patients), and bleeding resulting in hematoma requiring transfusion (1 patient). The most common complication of needle localization was asymptomatic pneumothorax (11/253 total patients; $4.3 \%)$ and was higher in patients with bullous emphysema (9/35 patients; $25.7 \%$ ). Of the 8 individuals who had unsuccessful needle localization, 7 had successful wedge resection in the area of methylene blue injection that included the nodule; 1 required segmentectomy for diagnosis. Completion lobectomy (154 VATS, 2 minithoracotomies) or VATS segmentectomy (18 patients) was performed in 174 individuals with a diagnosis of non-small cell carcinoma or carcinoid. The average length of hospital stay was 1.4 days for wedge resection, 1.9 days for VATS segmentectomy, 3.1 days for VATS lobectomy, and 4.9 days for minithoracotomy. Perioperative survival was $100 \%$.

Conclusions: Needle localization with hookwire deployment and methylene blue injection is a safe and feasible strategy to localize small, deep lung nodules for wedge resection and diagnosis. Multidisciplinary coordination between the thoracic surgeon and the interventional radiologist is key to the success of this procedure. (J Thorac Cardiovasc Surg 2018;155:2140-7)

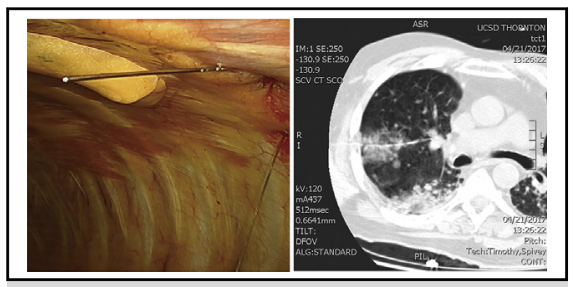

Hookwire complications of dislodgement (left) and migration (right).

\section{Central Message}

Needle localization with hookwire deployment and methylene blue injection is a safe and feasible method for localizing small, deep, or subsolid nodules before VATS

\section{Perspective}

Nodules that are small and deep within lung parenchyma, and have semisolid characteristics are often difficult to localize with VATS. We show that preoperative needle localization with hookwire deployment and methylene blue injection is a safe and feasible technique to localize nodules for wedge resection and diagnosis.

See Editorial Commentary page 2148.
More than 150,000 patients with pulmonary nodules are assessed by physicians each year in the United States, ${ }^{1}$ and approximately 80,000 individuals undergo thoracic surgery

From the ${ }^{\mathrm{a}}$ Division of Cardiothoracic Surgery, and ${ }^{\mathrm{b}}$ Department of Radiology, University of California, San Diego, Calif.

Funded by National Institutes of Health Grant R01 HL119543 to P.A.T.

Read at the 43rd Annual Meeting of The Western Thoracic Surgical Association, Colorado Springs, Colorado, June 24, 2017.

Received for publication June 18, 2017; revisions received Dec 14, 2017; accepted for publication Jan 7, 2018; available ahead of print Feb 15, 2018. each year to remove a solitary lung nodule. ${ }^{2,3}$ These numbers are increasing with the widespread adoption of lung cancer screening in at-risk populations. ${ }^{4}$ Increasingly,

\footnotetext{
Address for reprints: Patricia A. Thistlethwaite, MD, PhD, Division of Cardiothoracic Surgery, University of California, San Diego, 9300 Campus Point Drive, La Jolla, CA 92037-7892 (E-mail: pthistlethwaite@ucsd.edu). $0022-5223 / \$ 36.00$

Copyright (C) 2018 by The American Association for Thoracic Surgery https://doi.org/10.1016/j.jtcvs.2018.01.007
} 


\section{Abbreviations and Acronyms}

$\mathrm{CT}=$ computed tomography

$\mathrm{ENB}=$ electromagnetic navigational bronchoscopy

PET $=$ positron emission tomography

VATS $=$ video-assisted thoracoscopic surgery

Scanning this QR code will take you to a supplemental video for the article.

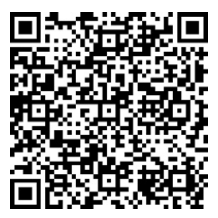

diagnoses are being sought for smaller and smaller pulmonary nodules, as well as lung lesions that have ground-glass appearance on computed tomography (CT) scanning.

Traditional options for lung nodule biopsy or excisional biopsy all have limitations. Percutaneous needle biopsy is associated with a risk of pneumothorax, is prone to sampling error, and is often not offered to patients with deep pulmonary nodules or nodules near major vascular structures. ${ }^{5}$ Although electromagnetic navigational bronchoscopic (ENB) biopsy has expanded the location of lung nodules that may be sampled, it is still an unreliable option for peripheral lesions or lesions at acute angles to the adjacent bronchial branch. Success rates vary from $38 \%$ to $86 \%$ for successful ENB biopsy. ${ }^{6,7}$ Excisional biopsy is often performed for diagnosis using video-assisted thoracoscopic surgery (VATS) techniques at the same time as definitive resection. However, the failure to visualize or palpate a lung nodule has been reported to occur in $48 \%$ to $54 \%$ of patients undergoing unguided VATS excision, thus necessitating conversion to thoracotomy. ${ }^{8,9}$ The conversion rate to thoracotomy has been reported to increase to $63 \%$ when the nodules are $10 \mathrm{~mm}$ or less in size or greater than $5 \mathrm{~mm}$ from the pleural surface. ${ }^{9}$ In addition, semisolid, ground-glass nodules are being referred for excisional biopsy at greater frequency because of the recognition that they may harbor areas of adenocarcinoma. Ground-glass lesions are often difficult to palpate, and a recent report suggests that $45 \%$ of groundglass lesions may be missed during VATS biopsy. ${ }^{10}$

We report our experience using needle localization with hookwire deployment and methylene blue injection, done under CT guidance, to localize small, deep lung nodules. We report our technique, localization success, operative results, and lessons learned over time. We show this technique provides a safe and feasible method of precisely finding small, deep nodules, allowing for parenchymal-sparing biopsies.

\section{MATERIALS AND METHODS Patients}

Retrospective review of patient data that comprise this article was approved by the University of California, San Diego Institutional Review Board in December 2016. The University of California, San Diego Institutional Review Board has waived the requirement for individual patient consent for this single institution analysis.

Between July 1, 2006, and December 30, 2016, 253 patients (86 male, 167 female; mean age, 67.3 years) were retrospectively identified as undergoing needle localization with hookwire placement and methylene blue injection before resection (Table 1). Patients were deemed candidates for needle localization if their nodules were deep $(>2 \mathrm{~cm}$ from the pleural surface) within the lung parenchyma, less than $1.2 \mathrm{~cm}$ in size, or growing or increasing in density on serial CT scanning. A total of 151 patients $(59.7 \%)$ had a history of smoking. Seventeen patients $(6.7 \%)$ had a previous ipsilateral thoracotomy. Preoperative workup included contrastenhanced CT scanning of the chest and upper abdomen, brain magnetic resonance imaging, positron emission tomography (PET) scanning, and pulmonary function testing. If PET avidity was seen in the mediastinum or mediastinal lymph nodes were greater than $1 \mathrm{~cm}$ on CT scanning, the patient underwent mediastinoscopy or endobronchial ultrasound-guided biopsy to rule out extrapulmonary disease before needle localization and resection. Seventy-one patients were directly referred from interventional radiologists at our institution for a combined needle localization-surgical biopsy approach. For these patients, the referring interventional radiologist thought that accurate core needle biopsy was limited because of nodule size or consistency, whereas needle and hookwire placement within 1 to $2 \mathrm{~cm}$ of the nodule was technically feasible.

\section{Needle Localization Planning and Procedure}

Before surgery, cases were reviewed by experienced interventional radiologists (A.C.P. and A.C.R.) and a thoracic surgeon (P.A.T.) to determine patient candidacy and the best approach for needle trajectory. Patients were excluded from consideration for needle localization for the following reasons: (1) the presence of pulmonary hypertension with pulmonary artery systolic pressure greater than $50 \mathrm{~mm} \mathrm{Hg}$, (2) limited pulmonary reserve with forced expiratory volume in 1 second less than $0.6 \mathrm{~L}$, (3) the inability of the patient to lie flat in the CT scanner for at least 30 minutes, and (4) the presence of lung nodules that would not be amenable to wedge resection for diagnosis because of invasion into major vascular structures. If a patient was excluded, he received current standard treatment, including continued observation of the nodule at 3- to 6-month intervals, attempted percutaneous needle biopsy or electromagnetic navigational bronchoscopy (ENB) biopsy, or excisional surgery (VATS or thoracotomy).

Nodule depth (in centimeters) from the pleural surface was obtained from CT measurement and defined as the distance between the nearest pleural surface and the edge of the nodule closest to the pleural surface. For needle localization procedure, see Video 1. Patients were lightly sedated and a scout CT scan was performed after placement of a grid to localize the skin entry site. After sterile prep and drape of the patient, $1 \%$ lidocaine was injected into the chest wall at the future site of needle insertion. Patients were asked to take small shallow breaths or hold their breath to determine which state was best to reduce the mobility of the nodule. A Hawkins III Hardwire Breast localization needle (20-gauge, $12.5 \mathrm{~cm}$ length) with built-in deployable 19-strand, 21-gauge hookwire with a barbed tip (Argon Medical Devices, Inc, Athens, Tex) was then inserted through the chest wall and advanced to the edge of the nodule using intermittent CT guidance. Once the needle was positioned, 0.3 to $0.6 \mathrm{~mL}$ of methylene blue was administered through the needle. The hookwire was then advanced out the tip of the needle and deployed so that the barb was immediately adjacent to the nodule. A second wire was then placed from a different trajectory using the same procedure. The needles were subsequently removed, leaving the hookwires in place. The portion of the wires outside the body were bolstered with gauze and taped to the 
TABLE 1. Patient and nodule characteristics

\begin{tabular}{|c|c|}
\hline & n $(\%)$ or mean \pm SD $($ range $)$ \\
\hline Total patients & 253 \\
\hline Age $(y)$ & $67.3 \pm 35.6(29-94)$ \\
\hline Gender (male) & $86(34)$ \\
\hline \multicolumn{2}{|l|}{ Smokers } \\
\hline Current & $45(17.8)$ \\
\hline Quit & $106(41.9)$ \\
\hline Never smoked & $102(40.3)$ \\
\hline Previous ipsilateral thoracotomy & $17(6.7)$ \\
\hline Previous extrathoracic cancer & $53(20.9)$ \\
\hline FEV1 & $71.3 \pm 20.7(34-129)$ \\
\hline DLCO & $60.9 \pm 18.6(31-88)$ \\
\hline Total nodules & 253 \\
\hline PET positive & $59(23.3)$ \\
\hline Nodule diameter $(\mathrm{mm})$ & $11.9 \pm 3(6-15)$ \\
\hline Depth from pleura (mm) & $33.7 \pm 8.1(19-49)$ \\
\hline \multicolumn{2}{|l|}{ Location } \\
\hline Right upper lobe & $68(26.9)$ \\
\hline Right middle lobe & $16(6.3)$ \\
\hline Right lower lobe & $59(23.3)$ \\
\hline Left upper lobe & $33(13.0)$ \\
\hline Left lower lobe & $77(30.4)$ \\
\hline \multicolumn{2}{|l|}{ Radiologic characteristics } \\
\hline Solid & $151(59.7)$ \\
\hline Ground glass & $16(6.3)$ \\
\hline Mixed solid-ground glass & $86(34.0)$ \\
\hline
\end{tabular}

$S D$, Standard deviation; $F E V I$, forced expiratory volume in 1 second; $D L C O$, carbon monoxide diffusing capacity; $P E T$, positron emission tomography.

skin, before patient transfer to the preoperative holding area or operating room.

\section{Surgical Procedure}

In the operating room, patients were anesthetized, placed in lateral decubitus position, and ventilated using a double-lumen endotracheal tube for single lung isolation. Wires were cut to a length of $1 \mathrm{inch}$ from the skin, and the wires prepped into the surgical field. Under thoracoscopic visualization, wires were then pulled completely into the pleural space. Gentle manipulation of the wire outside the lung provided haptic feedback and allowed for traction of the lung nodule in a direction that facilitated resection. VATS wedge resection was performed using an endostapler (Echelon Endostapler, Ethicon Inc, Cincinnati, Ohio). The specimen, encompassing the area of methylene blue and containing the full length of the 2 hookwires, was removed from the chest using a specimen retrieval bag (Ethicon Inc). Intraoperative frozen-section pathology was performed. If the frozen-section results were benign or showed metastatic disease with adequate negative margins, VATS was completed after excluding bleeding and air leak. Patients with primary non-small cell carcinoma of the lung underwent immediate lobectomy or segmentectomy, based on preoperative pulmonary function testing, as well as mediastinal lymphadenectomy for staging. Segmentectomy with mediastinal lymphadenectomy was chosen for patients with inadequate pulmonary function to tolerate a lobectomy or if the pathology showed adenocarcinoma in situ, minimally invasive adenocarcinoma, or carcinoid.

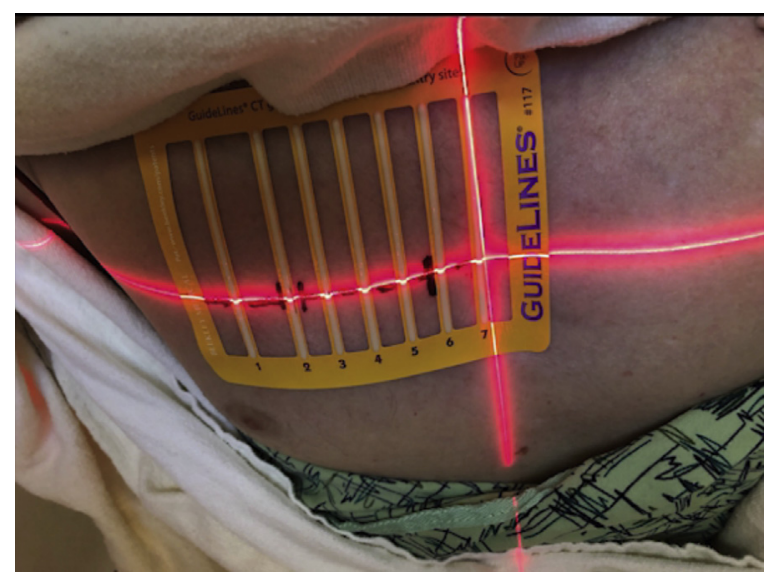

VIDEO 1. Needle localization for wedge resection of 2 right upper lobe lung nodules. Video available at: http://www.jtcvsonline.org/article/ S0022-5223(18)30055-2/fulltext.

\section{Statistical Analysis}

Data are presented as mean \pm standard deviation with range for continuous variables, unless otherwise indicated. Frequency and percentage are listed for categoric variables.

\section{RESULTS}

Needle localization was successful in 245 of 253 patients $(96.8 \%)$. The average nodule size was $11.9 \pm 3 \mathrm{~mm}$ (range, $6-15 \mathrm{~mm}$ ), and the average nodule depth was $33.7 \pm 8.1 \mathrm{~mm}$ (range, 19-49 mm) (Table 1). On CT scanning, 151 lesions $(59.7 \%)$ were considered solid, 86 lesions $(34.0 \%)$ were mixed solid and ground glass, and 16 lesions $(6.3 \%)$ were ground glass (Table 1). There was no exposure of radiation to the interventional radiologist, because CT imaging was done with the radiologist outside of the room. The mean dose of radiation for the last 100 patients was $5.89 \pm 4.3 \mathrm{mSv}$. The last 100 patients were billed $\$ 3250$ per case for needle localization (Current Procedural Terminology code: 10035).

Pathologic diagnosis included benign lesions (34), metastases (45), and primary lung malignancy (174) (Table 2). The benign lesions consisted of coccidioidomycosis nodules (22), tuberculosis (6), sarcoidosis (4), and radiation scar (2). The metastases consisted of colon (14), breast (12), larynx (9), rectum (4), kidney (3), thyroid (2), and soft palate (1). Primary adenocarcinomas were seen in 139 patients, with the following subclassification: invasive adenocarcinoma (121), variant of invasive adenocarcinoma (invasive mucinous adenocarcinoma) (2), minimally invasive adenocarcinoma (13), and adenocarcinoma in situ (3). Twenty-four patients had squamous cell carcinoma. Nine patients had large-cell carcinoma. Two patients had primary lung carcinoid. For benign and malignant lesions, the frequency of a positive margin after resection was zero. In 4 of 253 cases, the pathologist was unable to distinguish tissue origin of malignancy as an intraoperative 
TABLE 2. Lung nodule pathology

\begin{tabular}{lc}
\hline & $\mathbf{n}(\%)$ \\
\hline Total nodules & 253 \\
Benign lesions & $34(13.4)$ \\
Coccidioidomycosis & $22(8.7)$ \\
Tuberculosis & $6(2.4)$ \\
Sarcoidosis & $4(1.6)$ \\
Radiation scar & $2(0.8)$ \\
Metastatic lesions & $45(17.8)$ \\
Colon & $14(5.5)$ \\
Breast & $12(4.7)$ \\
Larynx & $9(3.6)$ \\
Rectum & $4(1.6)$ \\
Kidney & $3(1.2)$ \\
Thyroid & $2(0.8)$ \\
Soft palate & $1(0.4)$ \\
Adenocarcinoma of the lung & $139(54.9)$ \\
Invasive adenocarcinoma & $121(47.8)$ \\
Invasive mucinous & $2(0.8)$ \\
$\quad$ adenocarcinoma & \\
Minimally invasive & $13(5.1)$ \\
adenocarcinoma & \\
Adenocarcinoma in situ & $3(1.2)$ \\
Squamous cell carcinoma & $24(9.5)$ \\
Large cell carcinoma & $9(3.6)$ \\
Primary lung carcinoid & $2(0.8)$ \\
\hline
\end{tabular}

diagnosis. These patients underwent wedge resection with negative margins and were brought back to the operating room at a later date for definitive resection if the pathologic diagnosis was deemed to be non-small cell carcinoma.

Needle localization was associated with complications in 22 patients $(8.7 \%)$ (Table 3$)$. The most common complication was asymptomatic pneumothorax (11/253 patients; $4.3 \% ; 95 \%$ confidence interval $[\mathrm{CI}], 2.4-7.7)$, diagnosed by CT imaging after hookwire placement or by a rush of air with initial port placement, and was highest in individuals with panlobular emphysema or bullae greater than $2 \mathrm{~cm}$ in size (Table 4). One patient with both panlobular emphysema and bullae greater than $2 \mathrm{~cm}$ required chest tube placement for symptomatic pneumothorax before operation. No patient developed hemodynamic instability when anesthesia was induced and positive pressure ventilation instituted. Other complications included 1 wire (2 patients) or both wires (5 patients) falling out of the lung parenchyma before surgical visualization, wire migration more than $4 \mathrm{~cm}$ from the nodule (2 patients), and bleeding resulting in extrapleural hematoma around the subclavian vessels, necessitating transfusion in the interventional radiology suite and repair of the subclavian artery (1 patient) (Table 3). The 8 unsuccessful needle localization cases (8/ 253 patients; $3.2 \%$; $95 \%$ CI, 1.5-6.2) were due to both wires falling out before resection ( 5 patients), wire
TABLE 3. Procedural outcomes

\begin{tabular}{lc}
\hline & n $(\%)$ or mean \pm SD (range) \\
\hline Successful needle localization & $245(96.8)$ \\
Average time in interventional & $45 \pm 30(35-83)$ \\
radiology (min) & \\
Complications & $22(8.7)$ \\
Asymptomatic pneumothorax & $11(4.3)$ \\
Pneumothorax requiring chest tube & $1(0.4)$ \\
Single wire dislodgement & $2(0.8)$ \\
Double wire dislodgement & $5(2.0)$ \\
Wire migration & $2(0.8)$ \\
Extrapleural hematoma & $1(0.4)$ \\
\hline
\end{tabular}

$S D$, Standard deviation.

migration more than $4 \mathrm{~cm}$ from the nodule (2 patients), and extrapleural hematoma with bleeding (1 patient).

Of the 8 individuals who had unsuccessful needle localization, 7 had successful wedge resection in the area of methylene blue injection that included the nodule; 1 required blind segmentectomy for diagnosis. We found that delay in bringing the patient to the operating room after needle localization was associated with spread of methylene blue throughout lung parenchyma, resulting in a loss of effectiveness of this modality. We specifically found methylene blue as a marker of nodule location was ineffective in patients who had a delay of more than 2 hours between methylene blue injection and surgical visualization. In addition, all of the 7 patients who had wires fall out of the lung before operation had wait times in excess of 2 hours between needle localization and surgical visualization. This observation made us change our practice pattern to limit the wait between needle localization and operating start time to less than 1 hour for the last 95 patients. For the 2 individuals who had wire migration resulting in the wire tip more than $4 \mathrm{~cm}$ from the lesion on CT imaging, wires were removed under thoracoscopic visualization and wedge resection

TABLE 4. Pneumothorax incidence related to emphysema type

\begin{tabular}{lcc}
\hline & & $\begin{array}{c}\text { Patients with PTX } \\
\mathbf{n}=\mathbf{1 2}\end{array}$ \\
\cline { 3 - 3 } & $\begin{array}{c}\text { No. of patients } \\
\mathbf{n}=\mathbf{2 5 3}\end{array}$ & $\begin{array}{c}\mathbf{n} \text { (\% of each emphysema } \\
\text { type) }\end{array}$ \\
\hline Emphysema & 85 & $9(10.6)$ \\
Centrilobular & 64 & $2(3.1)$ \\
Panlobular & 7 & $5(71.4)$ \\
Paraseptal & 2 & $0(0)$ \\
Mixed & 12 & $2(16.6)$ \\
Bullous emphysema & 35 & $9(25.7)$ \\
Bullae $>2 \mathrm{~cm}$ & 6 & $2(33.3)$ \\
Bullae $<2 \mathrm{~cm}$ & 29 & $7(24.1)$ \\
No emphysema & 168 & $3(1.8)$ \\
\hline
\end{tabular}

PTX, Pneumothorax 

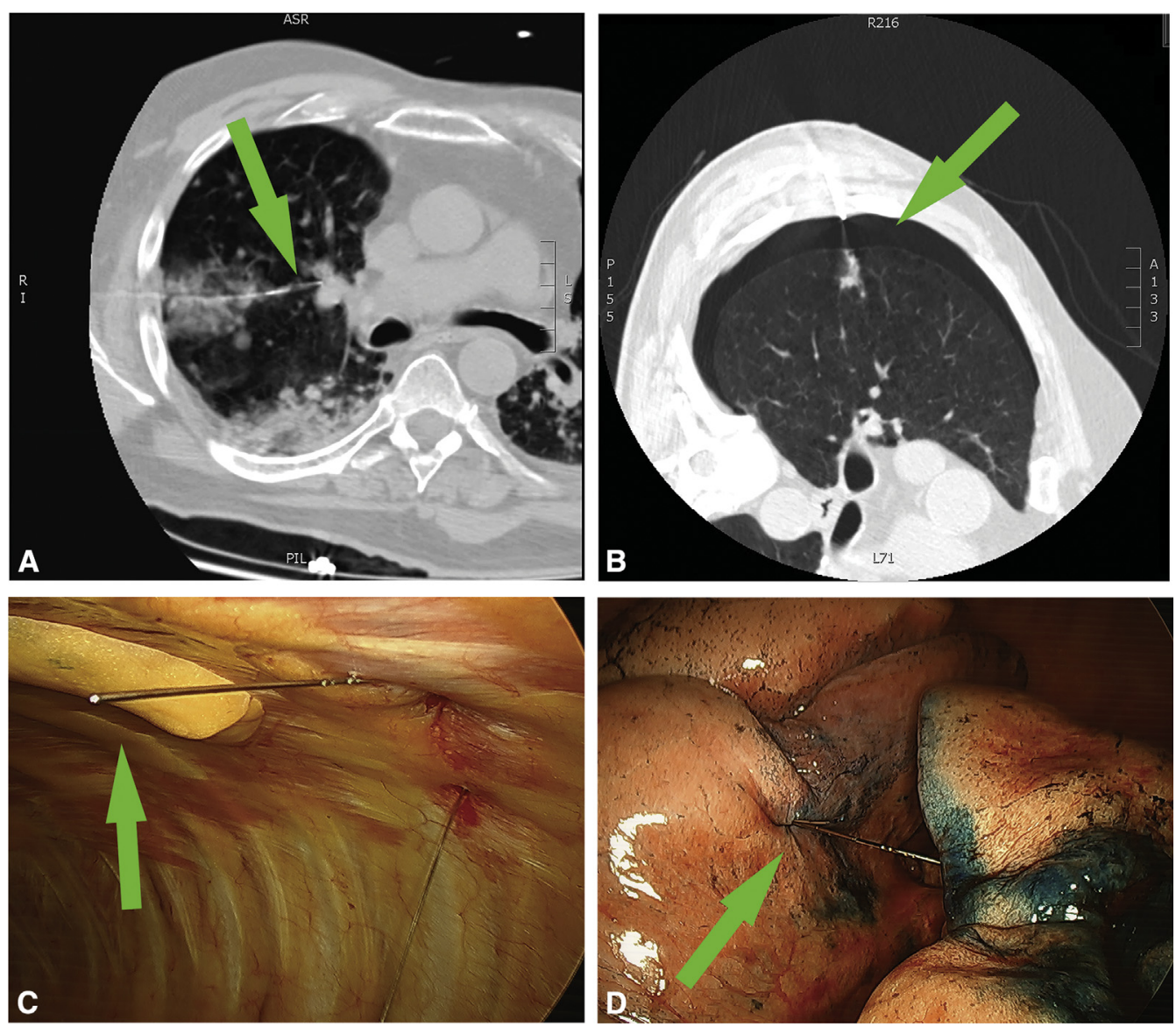

FIGURE 1. Complications. A, Unintended hookwire (green arrow) migration into the hilum, (B) pneumothorax (green arrow) after hookwire deployment, (C) hookwire (green arrow) dislodgement, and (D) unintended migration of hookwire through the fissure, with tip (green arrow) spearing the adjacent lobe.

was performed to encompass the entry sites of both wires (Figure 1).

For patients with non-small cell carcinoma (174/253 patients; $68.8 \%$ ), completion lobectomy was performed via a VATS approach for 154 individuals (150 patients immediately after frozen-section diagnosis, 4 patients at a later date because of inability of the pathologist to give a definitive frozen-section diagnosis) (Table 5). Two patients required lobectomy via minithoracotomy because of severe adhesions from previous thoracotomy. Eighteen individuals in whom lobectomy was precluded by limited pulmonary function or who had minimally invasive adenocarcinoma, adenocarcinoma in situ, or carcinoid underwent segmentectomy via a VATS approach immediately after frozensection diagnosis. The average length of hospital stay was 1.4 days for VATS wedge resection, 1.9 days for VATS segmentectomy, 3.1 days for VATS lobectomy, and 4.9 days for minithoracotomy. Postoperative complications included atrial fibrillation (3 patients; $1.2 \%$ ), air leak more than 4 days ( 8 patients; $3.2 \%$ ), cerebrovascular accident ( 1 patient; $0.4 \%$ ), and ileus ( 6 patients; $2.4 \%$ ). Perioperative survival was $100 \%$.

\section{DISCUSSION}

As lung cancer screening gains popularity and the quality of imaging modalities improves, the number of small pulmonary nodules detected is increasing. In addition, as systemic cancer therapies improve, there is an increasing role for surgical resection of pulmonary nodules in patients with a history of cancer, for both diagnostic and therapeutic purposes. VATS excision of lung nodules has become one of the main modalities for diagnosis of lung cancer and is often done before anatomic resection in the operating room. Despite widespread adoption of VATS excision of pulmonary nodules, there are cases in which precise localization is impossible because the nodule is neither visible nor palpable. These include semisolid lesions that have a large ground-glass to solid component, lesions deep within the lung parenchyma, and lesions in lung fields where previous surgery or radiation has been done. Our study shows that percutaneous needle localization with hookwire placement and methylene blue injection is a safe and feasible method of nodule localization for diagnostic VATS wedge resection. 
TABLE 5. Carcinoma of the lung: Surgical approach and outcomes

\begin{tabular}{lc}
\hline & n $(\%)$ or mean \pm SD (range) \\
\hline Operation type & \\
Primary lung cancer & $174(68.8)$ \\
$\quad$ VATS lobectomy & $154(60.9)$ \\
$\quad$ Minithoracotomy lobectomy & $2(0.8)$ \\
$\quad$ VATS segmentectomy & $18(5.5)$ \\
Metastatic lesions & \\
$\quad$ VATS wedge resection & $45(17.8)$ \\
Benign lung lesions & $34(13.4)$ \\
$\quad$ VATS wedge resection & \\
Mean length of hospital stay (d) & $1.4 \pm 1.9(1-3)$ \\
VATS wedge resection & $1.9 \pm 2.8(1-5)$ \\
VATS segmentectomy & $3.1 \pm 2.3(2-6)$ \\
VATS lobectomy & $4.9 \pm 2.9(3-6)$ \\
Minithoracotomy & \\
Postoperative morbidity & $3(1.2)$ \\
Atrial fibrillation & $8(3.2)$ \\
Air leak $>4$ d & $1(0.4)$ \\
CVA & $6(2.4)$ \\
Ileus & $253(100)$ \\
\hline Perioperative survival &
\end{tabular}

$S D$, Standard deviation; VATS, video-assisted thoracoscopic surgery; $C V A$, cerebrovascular accident.

Preoperative localization of lung nodules is not new. Multiple modalities, other than the one described in this article, are currently being evaluated to aid the thoracic surgeon in localizing small lung nodules. They fall into 3 major groups: (1) preoperative image-guided percutaneous placement of fiduciaries, radiotracers, and dyes; (2) preoperative or intraoperative ENB dye injection or fiducial placement; and (3) intraoperative ultrasound. Each modality has certain advantages and disadvantages. For example, for procedures done outside the operating room before surgery, the potential for miscommunication between teams should be weighed against the increased costs of operating room time, length of general anesthesia for the patient, and radiation exposure for a single team.

Percutaneous fiducial placement to localize lungs lesions has gained recent popularity. However, percutaneous fiducial placement under CT guidance is associated with a high risk of iatrogenic pneumothorax development of $13 \%$ to $47 \%,{ }^{11,12}$ with a recent study reporting a $25 \%$ pneumothorax rate. ${ }^{13}$ ENB is considered by some to be a less morbid alternative to accurately guide and deploy fiducial markers within the bronchial tree near endoscopically invisible peripheral lung nodules. However, previous studies using this technique report a $10 \%$ dislocation rate $^{14}$ and the requirement of general anesthesia for placement. ${ }^{15}$ Other complications from percutaneous or ENB fiducial placement include fiducial embolization and migration, parenchymal hematoma, and failure to retrieve markers at the time of operation. ${ }^{16}$

Dye injection to localize lung lesions that are difficult to find on the basis of size, depth, or density has been done by percutaneous CT-guided injection (as in this study), bronchoscopic-directed injection based on segmental anatomy, ${ }^{17,18}$ and ENB injection. ${ }^{19-21}$ All 3 methods have shown success; however, percutaneous CT-guided injection and ENB injection have demonstrated more precise localization over simple bronchoscopic-directed injection. ${ }^{22}$ Although difficult to compare different published series over time, reports suggest that greater dye particle size in the aqueous phase results in less dispersion of the dye through lung parenchyma over time. ${ }^{23}$ We chose and are familiar with methylene blue, which requires no extra equipment to visualize, but acknowledge that other contrast agents with larger molecular weight and larger particle size ${ }^{24}$ including indocyanine green, indigo carmine, iopamidol, and lipidiol may migrate slower into tissues (Table 6). The higher-density contrast agents iopamidol and lipidiol have been associated with allergic reaction to contrast medium, local inflammation at the site of injection, and contrast embolization. ${ }^{25,26}$

Finally, radiotracer-guided localization using gammaemitting radioisotopes (technetium 99) attached to large albumin molecules using CT-guided needle injection ${ }^{27,28}$ has the disadvantages of being highly facility-dependent because of the specific radiotracer and gamma probe used, with radiation exposure to surgeons and radiologists. ${ }^{29}$ Thoracoscopic ultrasonography with application of an ultrasound probe directly on the lung surface has been shown to be successful for localizing lung nodules. ${ }^{10,30}$ With this method, nodules are identified as a hypoechoic lesion with a hypoechoic shadow beneath the lesion. Unfortunately, ground-glass lesions are more difficult to localize by this modality, the procedure is highly operator-dependent, and localization requires a completely collapsed lung, often not possible in patients with emphysema. ${ }^{31}$

Our needle localization experience has been a multidisciplinary effort for more than 10 years. With this experience, we have learned multiple lessons that we hope will be helpful to other thoracic surgeons. First, we have stopped securing the hookwires at the skin with Hawkins clips, as we have seen more wire dislocation with the wires firmly locked in place at the skin. Rather, we coil and loosely cover the wires outside the skin, bolstering them with gauze pads to allow for movement with respiratory variation. Second, we find that methylene blue instillation is an effective marker for nodules; however, it cannot be seen on the lung surface in the case of deep nodules, and dye often spreads with time periods more than 2 hours between dye instillation and surgical visualization. Third, placement of hookwires at 90 degrees to surface of the lung closest to 
TABLE 6. Injectable imaging compounds

\begin{tabular}{lclll}
\hline Chemical compound & Size $\left(\mathbf{A}^{2}\right)$ & Method of excretion & Molecular formula & Additional equipment required \\
\hline Methylene blue & 43.9 & Renal & $\mathrm{C}_{16} \mathrm{H}_{18} \mathrm{ClN}_{3} \mathrm{~S}$ & None \\
Indocyanine green & 137 & Bile/GI tract & $\mathrm{C}_{43} \mathrm{H}_{17} \mathrm{~N}_{2} \mathrm{NaO}_{6} \mathrm{~S}_{2}$ & NIR fluorescence thoracoscope \\
Iopamidol & 188 & Renal & $\mathrm{C}_{17} \mathrm{H}_{22} \mathrm{I}_{3} \mathrm{~N}_{3} \mathrm{O}_{8}$ & Fluoroscopy \\
Lipiodol & - & Bile/GI tract & Unknown & Fluoroscopy \\
Indigo carmine & 189 & Renal & $\mathrm{C}_{16} \mathrm{H}_{8} \mathrm{~N}_{2} \mathrm{Na}_{2} \mathrm{O}_{8} \mathrm{~S}_{2}$ & None \\
\hline
\end{tabular}

A, Angstrom; GI, gastrointestinal; NIR, near-infrared. Chemical data from https://pubchem.ncbi.nlm.nih.gov/compound.

the lung nodule is preferable to limit parenchymal resection of lung tissue in the vicinity of the nodule. We have found that hookwires placed at acute angles to the surface of the lung are more likely to be dislodged before operation and make lung manipulation more difficult during VATS. Fourth, deployment of the hookwires should be immediately adjacent to the nodule and not within the nodule. "Pass point" deployment deep within the lung parenchyma (deeper than the deepest edge of the nodule) often leads to unnecessarily deep wedge resections that may distort the lobe, complicating anatomic resection. Although seeding of the needle tract with malignant cells is reported to be approximately $0.06 \%$ for percutaneous needle biopsy, ${ }^{32}$ we avoid direct needle or hookwire placement into the nodule, with wire deployment immediately adjacent to the nodule. Fifth, the entry sites for dislodged hookwires should be included in the resection specimen to prevent subsequent air leak if lobectomy is not performed. Finally, we realize that this technique requires a multidisciplinary approach, with back-and-forth communication between radiologist and surgeon, as well as coordination of patient time in the interventional radiology suite and operating room. In contrast to other techniques, our method does not require special imaging capability in the operating room (eg, near-infrared fluorescence thoracoscope, fluoroscopy) or other costly instruments (eg, endoscopic ultrasound probe, ENB system).

\section{Study Limitations}

We recognize that our study has the limitation of being a retrospective, nonrandomized analysis of a select cohort of patients. Nonetheless, to our knowledge, this is one of the largest series of needle localization thoracic operations performed to date. We tried to optimize patient selection by having a multidisciplinary team make the decision for performing needle localization with hookwire placement and methylene blue injection on each patient. We believe this combined approach reduces biopsy error and limits the amount of parenchyma resected for small, deeply located lesions by VATS. Our study demonstrates that preoperative CT-guided needle localization with hookwire placement and methylene blue injection is a feasible and safe procedure that allows for definitive excisional biopsy in $97 \%$ of patients. Advantages include a fast learning curve for the interventional radiologist, facile localization of nodules that might otherwise be difficult to identify during VATS for the surgeon, and immediate diagnosis with definitive resection under the same general anesthetic for the patient. Our technique encompasses all the characteristics of an ideal localization technique, including (1) a high accuracy rate, (2) a low morbidity rate, (3) minimal patient discomfort, (4) a short procedure time, (5) the ability to be applied to the whole lung field, (6) the use of equipment that is available in most hospitals, (7) limited radiation exposure time to patients, and (8) no radiation exposure to healthcare professionals. Although we consider each patient on a case-bycase basis, this technique is potentially applicable to most individuals who have small, deep, and semisolid nodules that would otherwise be difficult for the thoracic surgeon to find with thoracoscopy.

\section{CONCLUSIONS}

Needle localization is a safe and feasible method for targeted sublobar resection of nodules that are deemed difficult to localize. Further studies in the form of a randomized trial are warranted to assess and compare all the forms of localizing small lung nodules in terms of efficacy, patient comfort and risk, and cost.

\section{Conflict of Interest Statement}

Authors have nothing to disclose with regard to commercial support.

\section{References}

1. Jensen KW, Hsai DW, Seijo LM, Feller-Kopman DJ, Lamb C, Berkowitz D, et al Multicenter experience with electromagnetic navigation bronchoscopy for the diagnosis of pulmonary nodules. J Bronchol Interv Pulmonol. 2012;19:195-9.

2. 2015 United States Procedure Volumes Database. United States: Thomas Reuters; 2015. Available at: https://www.researchandmarkets.com/research/ q2jvn4/us_surgical. Accessed May 15, 2017.

3. National Inpatient Sample. Agency for Healthcare Research and Quality. Available at: www.hcup-us.ahrq.gov/nisoverview.jsp. Accessed May 15, 2017.

4. The National Lung Screening Trial Research Team, Aberle DR, Adams AM, Berg CD, Black WC, Clapp JD, Fagerstrom RM, et al. Reduced lug-cancer mortality with low-dose computed tomographic screening. N Engl J Med. 2011;365: 395-409.

5. Heerink WJ, de Bock GH, de Jonge GJ, Goren HJ, Vliegenthart R, Oudkerk M. Complication rates of CT-guided transthoracic lung biopsy: meta-analysis. Eur Radiol. 2017;27:138-48. 
6. Ost DE, Ernst A, Lei X, Kovitz KL, Benzaquen S, Diaz-Mendoza J, et al. Diagnostic yield and complications of bronchoscopy for peripheral lung lesions. Results of the AQuIRE registry. Am J Respir Crit Care Med. 2016;193:68-77.

7. Memoli JS, Nietert PJ, Silvestri GA. Meta-analysis of guided bronchoscopy for the evaluation of pulmonary nodules. Chest. 2012;142:385-93.

8. Finley RJ, Mayo JR, Grant K, Clifton JC, English J, Leo J, et al. Preoperative computed tomography-guided microcoil localization of small peripheral nodules: a prospective randomized controlled trial. J Thorac Cardiovasc Surg. 2015;149:26-32.

9. Suzuki K, Nagai K, Yoshida J, Ohmatsu H, Takahashi K, Nishimura M, et al. Video-assisted thoracoscopic surgery for small indeterminate pulmonary nodules: indications for preoperative marking. Chest. 1999;115:563-8.

10. Khereba M, Ferraro P, Duranceau A, Martin J, Goudie E, Thiffault V, et al. Thoracoscopic localization of intraparenchymal pulmonary nodules using direct intracavitary thoracoscopic ultrasonography prevents conversion of VATS procedures to thoracotomy in selected patients. J Thorac Cardiovasc Surg. 2012;144:1160-6.

11. Whyte RI, Crownover R, Murphy MJ, Martin DP, Rice TW, DeCamp MM, et al. Stereotactic radiosurgery for lung tumors: preliminary report of a phase I trial. Ann Thorac Surg. 2003;75:1097-101.

12. Pennathur A, Luketich JD, Heron DE, Abbas G, Burton S, Chen M, et al. Stereotactic radiosurgery for the treatment of stage I non-small cell lung cancer in highrisk patients. J Thorac Cardiovasc Surg. 2009;137:597-604.

13. Sharma A, McDermott S, Mathisen DJ, Shepard JO. Preoperative localization of lung nodules with fiducial markers: feasibility and technical considerations. Ann Thorac Surg. 2017;103:1114-20.

14. Harley DP, Krimsky WS, Sarkar S, Highfield D, Aygun C, Gurses B. Fiducial marker placement using endobronchial ultrasound and navigational bronchoscopy for stereotactic radiosurgery: an alternative strategy. Ann Thorac Surg. 2010;89:368-74.

15. Hagmeyer L, Priegnitz C, Kocher M, Shilcher B, Budach W, Treml M, et al. Fiducial marker placement via conventional or electromagnetic navigation bronchoscopy (ENB): an interdisciplinary approach to the curative management of lung cancer. Clin Respir J. 2016;10:291-7.

16. Sancheti MS, Lee R, Ahmed SU, Pickens A, Fernandez FG, Small WC, et al. Percutaneous fiducial localization for thoracoscopic wedge resection of small pulmonary nodules. Ann Thorac Surg. 2014;97:1914-9.

17. Endo M, Kotani Y, Satouchi M, Takada Y, Sakamoto T, Tsubota N, et al. CT fluoroscopy-guided bronchoscopic dye marking for resection of small peripheral pulmonary nodules. Chest. 2004;125:1747-52.

18. Sakamoto T, Takada Y, Endoh M, Matsuoka H, Tsubota N. Bronchoscopic dye injection for localization of small pulmonary nodules in thoracoscopic surgery. Ann Thorac Surg. 2001;72:296-7.

19. Hachey KJ, Digesu CS, Armstrong KW, Gilmore DM, Khullar OV, Whang B, et al. A novel technique for tumor localization and targeted lymphatic mapping in early-stage cancer. J Thorac Cardiovasc Surg. 2017; 154:1110-8.
20. Tay JH, Wallbridge PD, Larobina M, Russell PA, Irving LB, Steinfort DP. Electromagnetic navigation bronchoscopy-directed pleura tattoo to aid surgical resection of peripheral pulmonary lesions. J Bronchol Interv Pulmonol. 2016;23: 245-50.

21. Anayama T, Qiu J, Chan H, Nakajima T, Weersink R, Daly M, et al. Localization of pulmonary nodules using navigation bronchoscope and a near-infrared fluorescence thoracoscope. Ann Thorac Surg. 2015;99:224-30.

22. Lin MW, Tseng YH, Lee YF, Hsieh MS, Ko WC, Chen JY, et al. Computed tomography-guided patent blue vital dye localization of pulmonary nodules in uniportal thoracoscopy. J Thorac Cardiovasc Surg. 2016;152:535-44.

23. Abbas A, Kadakia S, Ambur V, Muro K, Kaiser L. Intraoperative electromagnetic navigational bronchoscopic localization of small, deep, or subsolid pulmonary nodules. J Thorac Cardiovasc Surg. 2017;153:1581-90.

24. National Center for Biotechnology Information. Pubchem Compound Database. Available at: https://pubchem.ncbi.nlm.nih.gov/compound: CID6099 (methylene blue), CID11967809 (indocyanine green), CID65492 (iopamidol), CID5284351 (indigo carmine). Accessed June 10, 2017.

25. Watanabe K, Nomori H, Ohtsuka T, Kaji M, Naruke T, Suemasu K. Usefulness and complications of computed tomography-guided lipiodol marking for fluoroscopy-assisted thoracoscopic resection of small pulmonary nodules: experience with 174 nodules. J Thorac Cardiovasc Surg. 2006;132:320-4.

26. Nomori H, Horio H, Naruke T, Suemasu K. Fluoroscopy-assisted thoracoscopic resection of lung nodules with lipiodol. Ann Thorac Surg. 2002;74:170-3.

27. Doo KW, Yong HS, Kim HK, Kim S, Kang EY, Choi YH. Needlescopic resection of small and superficial pulmonary nodule after computed tomographic fluoroscopy-guided dual localization with radiotracer and hookwire. Ann Surg Oncol. 2015;22:331-7.

28. Galetta D, Bellomi M, Grana C, Spaggiari L. Radio-guided localization and resection of small or ill-defined pulmonary lesions. Ann Thorac Surg. 2015; 200:1175-80.

29. Bellomi M, Veronesi G, Trifiro G, Brambilla S, Bonello L, Preda L, et al Computed tomography-guided preoperative radiotracer localization of nonpalpable lung nodules. Ann Thorac Surg. 2010;90:1759-65.

30. Rocco G, Cicalese M, La Manna C, La Rocco A, Martucci N, Salvi R. Ultrasonographic identification of peripheral pulmonary nodules through uniportal video-assisted thoracic surgery. Ann Thorac Surg. 2011;92:1099-101.

31. Mattioli S, D’Ovidio F, Daddi N, Ferruzzi L, Pilotti V, Ruffato A, et al. Transthoracic endosonography for then intraoperative localization of lung nodules. Ann Thorac Surg. 2005;79:443-9.

32. Tomiyama N, Yasuhara Y, Nakajima Y, Adachi S, Arai Y, Kusumoto M, et al. CTguided needle biopsy of lung lesions: a survey of severe complication based on 9783 biopsies in Japan. Eur J Radiol. 2006;59:60-4.

Key Words: lung cancer, image-guided surgery, tumor localization 